\title{
Zorro chevauche dans le temps et dans l'espace : escales en France et au Japon
}

\section{Alice Defacq}

\section{(2) OpenEdition}

Journals

\section{Édition électronique}

URL : http://journals.openedition.org/traduire/1565

DOI : 10.4000/traduire. 1565

ISSN : 2272-9992

\section{Éditeur}

Société française des traducteurs

\section{Édition imprimée}

Date de publication : 1 décembre 2018

Pagination : 63-74

ISSN : 0395-773X

\section{Référence électronique}

Alice Defacq, «Zorro chevauche dans le temps et dans l'espace : escales en France et au Japon »,

Traduire [En ligne], 239 | 2018, mis en ligne le 01 décembre 2018, consulté le 29 juin 2019. URL : http:// journals.openedition.org/traduire/1565; DOI : 10.4000/traduire.1565 


\section{Zorro chevauche dans le temps et dans l'espace : escales en France et au Japon(1)}

\section{Alice Defacq}

Le musical(2) aurait vu le jour entre 1866 et 1927. Les Américains reprennent alors des éléments de formes existantes, comme l'opérette et le vaudeville, pour créer leur propre genre : un spectacle alliant harmonieusement dialogue, chant et danse. Le musical connaîtra ensuite plusieurs développements au niveau des thèmes abordés et de la musique employée. II se décline ainsi en plusieurs sous-genres tels le musical comedy (Guys and Dolls), le musical play (Wicked), le musical drama (Fiddler on the Roof), le musical pour enfants (Shrek), le thriller musical (Sweeney Todd), le musical parody (Fifty Shades! The Musical Parody) et le jukebox musical (Mamma Mia!). Signalons aussi le revival, qui est " la reprogrammation d'une comédie musicale, avec une nouvelle mise en scène, de nouveaux décors et costumes, et une nouvelle distribution " (Perroux 2009 : 233). Un même book(3) peut alors connaître différentes versions. Deux raisons principales justifient ce phénomène : l'envie de faire de l'argent sans prendre trop de risques, car le coût de production d'un musical peut être onéreux ; et le désir de ne pas laisser le spectacle tomber dans l'oubli.

Telles sont les bases sur lesquelles nous greffons notre problématique, laquelle envisagera le revival comme un exercice de réécriture ayant pour objectif de s'adapter au pays cible. Pour ce faire, nous nous appuierons sur Zorro, produit en Angleterre en 2008. L'idée d'écrire un musical prenant pour point de départ le personnage fictif Zorro est venue du producteur Adam Kenwright. II fit appel à Stephen Clark pour la confection du book et aux Gipsy Kings pour la mise en musique. Le justicier masqué chevauchera ensuite à travers le monde entier, contraignant Clark à modifier le book en conséquence. Cette prolifération, véritable indicateur du succès du

(1) Je dédie cet article à Stephen Clark, un homme talentueux, passionné et d'une extrême gentillesse, parti trop tôt. Ce travail est ma modeste façon de lui rendre hommage et de le remercier pour nos échanges par courrier électronique.

(2) Nous employons le terme musical que les Français rendent habituellement par " comédie musicale ".

(3) Le livret d'opéra est généralement l'œuvre d'un seul librettiste tandis que le book est souvent le fruit d'une écriture à plusieurs mains, impliquant un dialoguiste, un compositeur et un lyricist. 
musical à l'étranger, souligne aussi la nécessité de le réécrire en fonction du pays d'accueil. Les changements apportés contribueront à améliorer le texte et à rectifier les erreurs remarquées lors des précédentes productions (Clark 2016a(4)). Cet article entend donc démontrer que le book est une "entité incomplète " (Bassnett 1991: 101) qui donne lieu à une série de changements et de négociations. Bien que le titre reste inchangé, le texte cache des réalités textuelles différentes. Afin de comprendre les raisons qui ont amené Clark à réviser le texte source, nous effectuerons une analyse comparée de trois aspects présents dans deux books de Zorro, ceux écrits pour Paris en 2009 et pour Tokyo en 2010, que nous abrégeons dorénavant par ZP et ZT.

Avant de débuter, quelques précisions s'imposent : 1) les books ont d'abord été rédigés en anglais puis adaptés en langue seconde ; 2) Clark nous a remis les versions 25.6 (écrite pour Paris) et 27.9 (écrite pour Tokyo) ; 3) Éric Taraud adapte le texte français (désormais T), et Yoko Sakai (dialogue) signe avec Naoyuki Matsuda (lyrics) le texte japonais (désormais SM) ; 4) Taraud nous a confié l'adaptation française, Matsuda "ZorroLatestVersion " (reçue par Toho Production), la version 28.0 (utilisée sur scène) et "ZorroJapanChrisAct1_1118 " / "ZorroJapanChrisAct2_1118" (rédigée pour Christopher Renshaw(5)). Même si notre étude repose sur un seul book, nous espérons toutefois dresser quelques conclusions et montrer combien l'écriture du musical peut dépendre du pays cible.

Commençons par le découpage similaire des deux books : deux actes divisés en huit et onze scènes. Un prologue de flamenco ouvre les scripts, et la chanson "Bamboleo, Bambolea " les clôture. La majorité des actes s'ouvrent avec des didascalies précisant le jeu des acteurs et la mise en scène. Clark donne ainsi des indications temporelles et scénographiques comme The town square. Dawn [La place du village. Aube(6)] (acte 1, scène 6). Toutes les notes scéniques sont identiques, à l'exception d'un changement toponymique dans la scène 5 de l'acte 2 : nous sommes dans une quarry (carrière) dans ZP et une mine shaft (坑道(7), soit puits de carrière) dans ZT. Cet endroit joue un rôle majeur puisque c'est ici qu'est enfermé Alejandro, le père de Zorro. Désireux de rendre le lieu plus propice à la claustrophobie, Renshaw demande au scénographe de réviser le décor initial tout en gardant les matériaux accumulés lors des précédentes versions afin de s'assurer de leur impact sur le spectateur cible. II opte alors pour d'énormes morceaux de bois susceptibles de délimiter la trappe, le tout éclairé par une lumière très étrange. Clark avoue que ce puits de carrière lui donna même la chair de poule (2016c). Mission réussie! Nous touchons ici l'un des problèmes posés par la mise en acte d'un book

(4) Les explications de Clark, comme celles de Matsuda et de Taraud ci-après, proviennent de nos échanges personnels indiqués en bibliographie.

(5) Ce book a été spécialement conçu à l'intention du producteur, afin qu'il puisse suivre le jeu des acteurs japonais sur scène. II renferme le texte anglais, la traduction japonaise en rōmaji et la traduction littérale anglaise (ou backtranslation). Nous l'utilisons ici comme version de référence.

(6) Traduction de cette didascalie et de tous les extraits suivants par Taraud.

(7) Traduction de cette didascalie et de tous les extraits suivants par Sakai et Matsuda. 
pour différents publics. II faut non seulement prendre en compte leurs possibles réactions, mais aussi les atteindre au plus profond d'eux-mêmes. Le processus de production permet alors de modifier et de perfectionner le décor original afin de produire une dimension spatiale satisfaisante aux yeux de chacun.

Cette différence est minime, car elle ne modifie pas le déroulement de l'histoire. D'autres écarts auront un impact plus important, notamment la distribution, à savoir le nombre de personnages. Dans ZP et ZT, nous retrouvons les sept acteurs principaux (le vieux gitan, Diego de la Vega, alias Zorro, Ramón, Luisa, Inéz, Garcia et Alejandro) et les secondaires (le prêtre, le soldat, les paysans et les gitans). Mais, dans ZP, nous faisons la connaissance du maître d'armes français et de Gomez alors que dans ZT, ces derniers cèdent leur place à une voyante et trois enfants (Ramón, Diego, Luisa). Les multiples représentations du musical vont permettre de découvrir que certains personnages sont inutiles et seront par conséquent supprimés (Clark 2016b). Prenons l'exemple du maître d'armes français : plutôt que de dire au spectateur que Zorro manie brillamment l'épée, Clark préfère le montrer. II est indéniable que les "matrices textuelles de "représentativité" " (Ubersfeld 1996 : 16), c'est-à-dire les éléments non verbaux optiques et acoustiques que sont les gestes, les costumes, la voix et les expressions faciales, constituent une somme d'informations suffisantes pour la bonne compréhension complète du musical. Clark ne supprime pas le message initial, mais élimine tout ce qui ralentit l'action et se voit alors contraint d'opérer un véritable travail de réécriture.

Poursuivons avec la relation fraternelle entre le protagoniste Diego et l'antagoniste Ramón qui fut beaucoup plus problématique. II convient de préciser pour la suite de cette étude qu'ils sont frères de sang dans ZP mais se battent à la fin. Dans cette dernière scène, la mort accidentelle de Ramón suscitera la réaction de Toho Production. En effet, dès réception du script, la société s'attendait à lire les aventures d'un héros combattant le mal et non celles d'un homme tuant son frère par inadvertance. Le public japonais n'aurait donc pas pu expliquer la manière étrange dont Ramón décède (Matsuda 2017a). Par ailleurs, Clark confesse que les Japonais n'auraient pas accepté qu'un homme puisse tuer son propre frère (2016a). Comme cette scène finale posait d'évidents problèmes, notamment à cause des divergences dans les conventions culturelles, Clark fit donc de Diego et Ramón des frères adoptifs. II dut par conséquent tordre ZP pour insérer la nouvelle relation dans ZT :

\begin{tabular}{|c|c|c|c|c|c|}
\hline $1 \mathrm{a} \mid$ & RAMÓN & $\begin{array}{l}\text { But your cause was noble... to } \\
\text { bring back my dear brother( }(8) \\
\text { to the family home. (ZP, } 24)\end{array}$ & $1 c$ & RAMÓN & $\begin{array}{l}\text { Mais ta cause était noble... } \\
\text { Ramener mon cher petit frère } \\
\text { à la maison. }(T, 33)\end{array}$ \\
\hline $1 b$ & Ramón & $\begin{array}{l}\text { But thank you... What a relief } \\
\text { to have the prodigal son back } \\
\text { at the family home. (ZT, 74) }\end{array}$ & $1 d \mid$ & ラモン & $\begin{array}{l}\text { だがありがとう…放蕩息子の帰還 } \\
\text { に手を貸してくくれて。 } \\
\text { (SM, 74) }\end{array}$ \\
\hline
\end{tabular}

(8) Les caractères gras ont été ajoutés pour signaler les problèmes étudiés dans le texte. 
2a $\mid$ Diego He loved both of us. He gave us 2c everything he could.

RAMÓN All he gave me was a brother. A brother who stole my life.

DiEgo No. He gave you a brother who understands you. A brother you can trust. (ZP, 82)

2b | DIEGo He loved both of us. He gave us everything he could.

RAMÓN And you were as blind as he was... I wanted you to know what it was like... what it was like to lose a father!

Diego But I loved you. We were like brothers.

RAMÓN But we weren't brothers!

Diego Ramón. We can leave the past behind. I understand you. I love you. Please. Trust me... (ZT, 110-111)
DiEgo C'est faux. II nous a aimés tous les deux. II nous a donné tout ce qu'il a pu.

RAMÓN Tout ce qu'il m'a donné c'est un frère. Un frère qui m'a volé ma vie.

DiEgo Non. II t'a donné un frère capable de te comprendre. Un frère en qui tu peux avoir confiance. (T, 101)

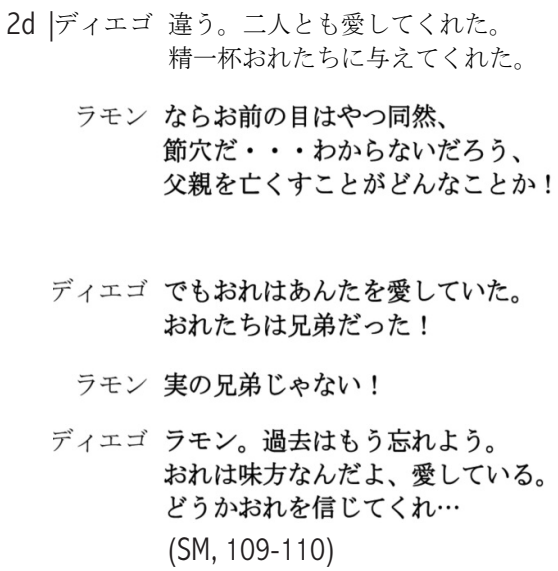

La mise en regard de ZP et ZT permet de noter que les réagencements syntaxiques s'opèrent à deux niveaux : d'une part, le remplacement de my dear brother (1a) par une locution appropriée, soit the prodigal son (1b); d'autre part, la réécriture complète des échanges entre les deux acteurs (2b). Sans conteste, Clark offre des alternatives qu'il suppose - à juste titre - adéquates pour défroisser au mieux les difficultés les plus délicates. Les solutions proposées s'imbriquent parfaitement dans le nouveau book et produisent le même effet escompté : deux hommes qui entretiennent une relation complexe. II est clair que ce remaniement textuel n'a pas posé de véritable problème à Clark. Sa tâche s'est quelque peu compliquée lorsqu'il s'est attelé aux lyrics puisqu'il devait préserver la métrique de départ, soit les temps forts et faibles musicaux, et la prosodie initiale ou plus largement la structure syllabique. Cet aspect se subdivise en "l'accent tonique et le poids. Le vocable "poids" [...] renvoie à la "valeur" de la syllabe déterminée dans la ligne mélodique et son rapport avec les autres syllabes " (Apter et Herman 2016 : 17)(9). Les modifications apportées dans la phrase musicale doivent donc être le plus naturelles possible. Le chant « Hope " illustre ces quelques difficultés :

(9) 'Stress (accent) and burden. By 'burden,' [...] is meant the 'weight' of a syllable that determines both the minimum time required to sing it and the relative time it requires in comparison with the syllables beside it". 


\begin{tabular}{|c|c|c|c|}
\hline $3 a \mid$ & $\begin{array}{l}\text { DIEGO How can I believe } \\
\text { My brother could destroy } \\
\text { All my father lived for? [...] } \\
\text { By my father's love I swear } \\
\text { We were free from fear } \\
\text { But the day I left these people } \\
\text { (ZP, 19-20) }\end{array}$ & 3c I Diego & $\begin{array}{l}\text { Comment accepter } \\
\text { Que mon frère ait détruit } \\
\text { Le rêve de mon père }[\ldots] \\
\text { Toi ma terre qui désespère } \\
\text { Je viens vers toi } \\
\text { En partant vers d'autres mondes } \\
(\mathrm{T}, 27)\end{array}$ \\
\hline $3 b \mid$ & $\begin{array}{l}\text { DiEGO How can I believe } \\
\text { That one man could destroy } \\
\text { All my father lived for? [...] } \\
\text { By my father's love I swear } \\
\text { We were free from fear } \\
\text { But the day I left these people } \\
\text { (ZT, 60-62) }\end{array}$ & 3d | ディエゴ & $\begin{array}{l}\text { 荒れ果てた この大地に } \\
\text { 希望の国を } \\
\text { 築くこと 父の夢 }[\cdots] \\
\text { 戦おう } \\
\text { 父の夢 かなえるきっと } \\
\text { 生まれ育った故郷 } \\
\text { (SM, 60-62) }\end{array}$ \\
\hline
\end{tabular}

Comme la préservation de my brother (3a) était bien évidemment impossible en (3b), il fut convenu de le remplacer par un substantif respectant la métrique musicale. Plus précisément, il fallut choisir trois syllabes susceptibles de s'installer sur la musique initiale et d'évoquer la relation établie dans ZT. En raison des restrictions rythmiques, Clark remania ainsi cette phrase et choisit that one man (3 syllabes). Quant aux deux lignes suivantes, à l'apparence innocente, elles ont en réalité donné lieu à de nombreuses négociations. Matsuda raconte qu'elles ne figuraient pas dans le book original de Londres en 2008 (version 22.5) et qu'elles ont été insérées, entre les lignes mélodiques 30 et 31, dans le book de Paris en 2009 (version 25.6). Elles sont ensuite gardées dans les books de Moscou en 2010 (version 27.7) et "Zorro LatestVersion " (pour Tokyo, datant précisément du 10 juin 2010). Puis elles sont supprimées de la version 28.0 (utilisée pour la scène japonaise et datant du 12 octobre 2010). Cela veut donc dire que la coupure a été effectuée entre le 10 juin et le 12 octobre 2010. Et, chose étonnante, ces deux lignes ont réapparu dans "ZorrolapanChrisAct1_1118 " (book conçu pour Renshaw datant du 10 novembre 2010) (Matsuda 2017c). L'adaptateur nous confie qu'elles " ont été écrites non pas à la machine mais à la main! Je les ai donc rajoutées pendant les répétitions " (2017b)(10). Sans savoir exactement pourquoi elles ont resurgi entre le 12 octobre et le 10 novembre 2010, Matsuda suppose que Renshaw ne voulait pas de blanc à ce moment-là, car la musique continuait. Par ailleurs, Taraud se souvient qu'ı il a été dit que la chanson ne "décollait" pas comme on pouvait l'espérer. Notamment à la fin. Michael Dixon, le superviseur musical, a inséré cette ligne mélodique supplémentaire vers le début de la chanson, durant les répétitions. II s'agissait musicalement de donner un aperçu mélodique de ce que

(10) "These two lines were inserted, not printed but written in my handwriting! This means I made the additional lyric for these two lines at the rehearsal." 
serait la toute fin de la chanson (le passage dans les aigus) " (2018). Cet exemple suffit à mettre en évidence le fait que le book renferme " un nombre virtuellement indéfini de mises en scène " (Regattin 2004 : 164). Le processus de production permet de procéder à des ajustements puisque le book est adapté - à l'occasion et ponctuellement, il est vrai - en fonction de la scène qui l'accueille. C'est avant tout la traduction d'une performance qui sera appréciée peu ou prou par le public cible. Signalons, pour être complet, qu'après avoir été frères de lait puis meilleurs amis, Diego et Ramón redeviennent frères de sang dans la version écrite pour Atlanta en 2013. Luisa endosse alors le rôle de la méchante, celle qui tue! (Clark 2016b). Cette solution a permis de maintenir la rivalité grandissante entre les deux hommes et d'esquiver le fratricide et les réactions voire déceptions de la salle.

Terminons par le mélange codique qui renvoie, dans son extension la plus large, à " tout type d'interaction entre deux ou plusieurs codes linguistiques différents dans une situation de contact des langues. Une telle définition englobe l'emprunt [...] et l'alternance codique " (Moreau 1997 : 207). II est de coutume d'associer le transfert linguistique à une lacune puisqu'il implique l'importation pure et simple de mots sans changement de graphie. Prenons l'exemple de tacos qui n'a pas d'équivalent en traduction et qui devient par conséquent partie intégrante de la langue d'accueil. Quant à l'alternance codique, elle correspond à l'emploi simultané de deux langues dites autonomes. Elle reflète une identité sociale parce qu'elle est la résultante de mélanges de populations parlant des langues différentes. Zorro brasse ainsi les langues angloaméricaine et espagnole. Rappelons que le musicalse déroule dans El Pueblo de Los Angeles, village fondé en 1781 durant la colonisation espagnole de la Haute Californie. Le traité de Guadeloupe Hidalgo (1848) mettra fin à la guerre américano-mexicaine (1846-1848) amenant le Mexique à céder une partie de son territoire, dont la Haute Californie, aux États-Unis. L'espagnol fait ainsi partie de l'héritage d'El Pueblo et y est, de nos jours, aussi fortement ancré.

4a | RAMÓN My boots. There is something on my boots. Clean them.

GARCIA Si, mi Capitán! (ZP, 61)

4b | Ramón My boots. There is something on my boots. Clean them.

GARCIA Si, mi Capitán! (ZT, 49) 4c | RAmón Mes bottes. II y a quelque chose sur mes bottes. Essuie-les.

GARCIA Si, mi Capitán ! (T, 78)

4d | ラモン 俺の靴に。靴に何かついちまった。 掃除しろ。

ガルシアシ、閣下。(SM, 49) 


\begin{tabular}{|c|c|c|c|}
\hline $5 \mathrm{a} \mid$ & $\begin{array}{l}\text { DIEGO Come and leave your hacienda } \\
\text { Oh señoras y señores } \\
\text { For now you can surrender } \\
\text { To the rhythm of guitars }[\ldots] \\
\text { Baila baila baila baila } \\
\text { Baila baila baila me } \\
\text { Esta rumba tan gitana } \\
\text { Que yo siempre cantaré } \\
\text { (ZP, 5-6) }\end{array}$ & $5 c \mid$ Diego & $\begin{array}{l}\text { Comme je sais d'avance vos } \\
\text { faiblesses } \\
\text { On a la nuit pour y gouter } \\
\text { Osez quitter vos haciendas } \\
\text { Tant de rêves à caresser }[. . .] \\
\text { Baila baila baila baila } \\
\text { Baila baila baila me } \\
\text { Esta rumba tan gitana } \\
\text { Que yo siempre cantaré }(\mathrm{T}, 11)\end{array}$ \\
\hline $5 b \mid$ & $\begin{array}{l}\text { DIEGO Come and leave your hacienda } \\
\text { Oh señoras y señores } \\
\text { For now you can surrender } \\
\text { To the rhythm of guitars [...] } \\
\text { Baila baila baila baila } \\
\text { Baila baila baila me } \\
\text { Esta rumba tan gitana } \\
\text { Que yo siempre cantaré } \\
\text { (ZT, 18-19) }\end{array}$ & $5 d$ |ディエゴ & 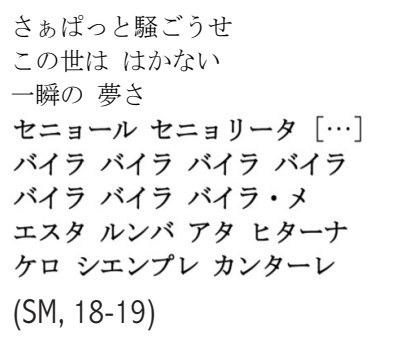 \\
\hline
\end{tabular}

Nous effleurons ici quelques problèmes posés par le recours au mélange codique. Comme il est impossible de jouer un book entièrement écrit en espagnol devant un public français ou japonais, à moins d'utiliser le surtitrage(11), Clark doit se montrer attentif à l'enjeu et à l'impact de la langue étrangère sur le public. Son usage découle alors de deux choix :

1) l'insertion sporadique de termes espagnols dans le dialogue, pour l'enrichir, illustrer un besoin de décentrement et faire " couleur locale " (Van Hoof 1989: 137). L'exemple du Si, mi Capitán (4a) reflète l'identité et les compétences polylectales du sergent. Notons que cette locution donnera lieu à deux traductions quelque peu différentes : en (4c), elle est gardée telle quelle, car le spectateur français reconnaît indiscutablement le substantif " capitaine ", tandis qu'en (4d), elle est partiellement rendue. La traductrice a effectivement éprouvé le besoin de conserver si (シ) et de traduire Capitán (閣下) afin d'en faciliter, sans doute, sa réception par le public et insérer une pointe d'humour. II reste à signaler que l'acteur japonais utilisait ce mot espagnol intelligemment pour faire rire l'audience (Matsuda 2017b). Le " verbocorps "(12) du comédien compensait donc la traduction partielle de Sakai, car " souvent le dire compte autant sinon plus que le dit " (Israël 1991 : 19). II faut effectivement produire un book qui puisse être joué en toute sincérité sur scène. Cette remarque a le mérite de rappeler que

(11) Traduction qui « se place au-dessus d'une scène ou au dos d'un fauteuil pour rendre une pièce de théâtre ou un opéra " (Gambier 2004 : 3).

(12) " Le discours [...] porte en lui des gestes, des mouvements, des intentions. [...] C'est donc ce que l'on nomme le verbo-corps, c'est-à-dire l'alliance du geste et du mot " (Péran 2011: 165). 
le book porte une "performability (jouabilité) [qui] renvoie à l'idée de jeu (du comédien) et de représentation au sens de réalisation scénique " (Gregory $2010: 7$ ) ;

2) le louvoiement de l'anglais et de l'espagnol dans les numéros musicaux. Signalons à ce stade que $50 \%$ des chants du musical ont été exclusivement écrits pour Zorro comme "Libertad " et que les $50 \%$ restants viennent du répertoire des Gipsy Kings tel que "Baila, baila me ". La décision de conserver les lyrics (5a) relève du choix de Clark de sélectionner les chants qui ont fait le succès du groupe mythique pour les insérer ensuite dans son book. En somme, " peu importe les intentions de l'artiste ou de l'auteur-compositeur à opter pour des lyrics bilingues, les effets obtenus peuvent varier considérablement en fonction des antécédents du public " (Davies et Bentahila 2008 : 261)(13). Leur conservation ou leur suppression ne se résument donc pas à la question de savoir s'il faut ou non tout garder, mais elle met en jeu un réseau de problèmes annexes comme le public cible et l'intérêt de ces lyrics dans l'intrigue. Clark navigue ainsi entre logocentric et musicocentric (Low 2017 : 10)(14) puisque les chants appréciés pour leur musique demeurent intacts, et que ceux jugés indispensables pour l'intrigue sont adaptés. Taraud renchérit en disant que " seuls certains refrains extrêmement connus ont été gardés en espagnol. [...] On considère que le public aime entendre le gimmick tel qu'il l'a toujours connu. Ça ne me dérange pas. Surtout dans un spectacle de pur divertissement comme Zorro " (2017).

Passant outre une utilisation quasi similaire de l'espagnol dans les deux books, nous nous intéressons davantage à son altérité. Clark met en place deux stratégies d'écriture non aléatoires, mais en accord avec les exigences du pays cible : 1) l'utilisation de l'espagnol dans ZP et sa traduction anglaise dans ZT ; 2) l'insertion de l'espagnol dans ZP et son gommage dans ZT. Regardons la première disparité :

6a | GARCIA Take aim! [...]

Luisa and Inéz enter, followed by Joaquin, the Old Gypsy and a few other Gypsies. The soldiers see them.

SOLDIER Extranjeros! (ZP, 15)

6b | GarCla Take aim! [...]

Luisa and Inéz enter, followed by Joaquin, the Old Gypsy and a few other Gypsies. The soldiers see them. 6c| GarCIA Et feu! [...]

Luisa et Inéz entrent, suivies par Joaquín, le vieux gitan et quelques bohémiens. Les soldats les voient.

SOLDAT Extranjeros! (T, 22)



ルイ其、イネス、ホアキン、老ジプシー ほか数人のジプシーが登場。

兵土たち彼らに気づく。

SOLDIER Strangers! (ZT, 47-48)

兵士 ヨソモンだ! (SM, 47-48)

(13) "Whatever the intentions of the artist or songwriter in opting for bilingual lyrics are, the effects achieved may vary considerably, depending on the audience's background".

(14) 'We can apply the term 'logocentric' to actual songs where the words matter more, and 'musicocentric' to the others". 
Le substantif extranjeros (6a), qui se rapproche du substantif "étranger ", est supprimé et remplacé par sa traduction anglaise, strangers (6b). II s'agit d'une utilisation par reconnaissance, car le français et l'espagnol entretiennent des rapports étroits par leur appartenance à la même famille, celles des langues romanes. Dans ZP, Clark insère des mots espagnols fortement liés au français et utilise, en toute logique ou presque, leur équivalent anglais dans ZT. En (6d), il est toutefois intéressant de préciser que Sakai rend strangers par ヨソモンだ [yoso mon] et non よそ者 [yoso mono], qui est sa traduction japonaise : ょそ [yoso] signifie " autre " ou " différent " et 者 [mono] " gens ". La traductrice choisit donc d'insérer une certaine subtilité puisqu'elle omet le " 0 " final de 者 [mono] et écrit le terme en katakana, qui est, précisons-le, la transcription japonaise des mots étrangers (Matsuda 2018). Concrètement, Sakai est extrêmement sensible aux attentes du public cible et propose divers subterfuges pour maintenir le dépaysement interne de départ.

Cette première stratégie d'écriture est cohérente et rigoureuse. La deuxième technique nous paraît plus draconienne puisqu'elle induit le gommage massif de l'espagnol dans ZT :

\begin{tabular}{|c|c|c|c|}
\hline \begin{tabular}{l|l|l} 
PEOPLE &
\end{tabular} & $\begin{array}{l}\text { - Dónde está Don Alejandro! } \\
\text { Escúchanos por favor! } \\
\text { - Zorro is on our side! Esto es } \\
\text { cosa del demonio! } \\
\text { - We want justice! Esto es cosa } \\
\text { del demonio! } \\
\text { - Dónde está el Alcade! We want } \\
\text { Alejandro! } \\
\text { - Vamos! Ven! Dónde está el! } \\
\text { - Que salga el Alcade! Esto es } \\
\text { Diabolico! } \\
\text { - Que salga Alejandro! Give us } \\
\text { Alejandro! } \\
\text { - No lo vamos a aceptar! (ZP, 47) }\end{array}$ & $7 c \mid$ & $\begin{array}{l}\text { EUPLE - Dónde está Don Alejandro! } \\
\text { - Escúchanos por favor! } \\
\text { - Zorro est avec nous ! Esto es } \\
\text { cosa del demonio! } \\
\text { - Nous voulons la justice ! Esto } \\
\text { es cosa del demonio! } \\
\text { - Dónde está el Alcade! Nous } \\
\text { voulons Alejandro! } \\
\text { - Vamos! Ven! Dónde está el! } \\
\text { - Que salga el Alcade! Esto } \\
\text { es Diabolico! } \\
\text { - Que salga Alejandro! Rendez- } \\
\text { nous Alejandro! } \\
\text { - No lo vamos a aceptar! (T, 61) }\end{array}$ \\
\hline $7 \mathrm{~b} \mid$ PeOPLE & $\begin{array}{l}\text { - Where is Don Alejandro! } \\
\text { - Zorro is on our side! } \\
\text { - We want justice! } \\
\text { - We want Alejandro! } \\
\text { - Give us Alejandro! } \\
\text { - Bring him here! Give us justice! } \\
\text { (ZT, 4) }\end{array}$ & $7 d$ & $\begin{aligned} \text { 群集 - アレハンドロはどこだ? } \\
\text { - ゾロはおれたちの味方だ! } \\
\text { - 正義を我らに! } \\
\text { - アレハンドロを返せ ! } \\
\text { - アレハンドロ、返せ ! } \\
\text { - つれて来い! 正義はどうした！ } \\
\quad \text { (SM, 4) }\end{aligned}$ \\
\hline
\end{tabular}

L'espagnolité, bien marquée en (7a), se fait fuyante en (7b). Le sens des répliques reste certes le même, mais perd une certaine saveur. Même si cette amputation a comme conséquence d'appauvrir la coloration espagnole du book, elle n'entraîne aucune rupture narrative. En outre, 
Clark expliquait que cette langue seconde aurait été complètement inutile et même aliénée, car l'espagnol est trop éloigné de la langue japonaise (Clark 2016d). À cet égard, Matsuda ajoute qu'il a régulièrement dû adapter les lyrics afin de réduire le sentiment inconfortable ressenti par les Japonais dès qu'ils entendent des mots étrangers qu'ils ne comprennent pas (2017b). II nous rappelle alors que le book est avant tout destiné à la scène et qu'il est préférable d'amoindrir la saveur espagnole plutôt que de perdre le spectateur avec une langue qui n'est pas la sienne. Bien entendu, les objets théâtraux comme le décor, la tenue des comédiens et les accessoires compensent ce gommage puisque leurs " fonctions utilitaires ou métonymiques sont évidentes " (Ubersfeld 1978 : 199, cité dans Koustas 1988 : 134). Ils savent suggérer l'emplacement du lieu de l'action et ils donnent aux personnages qui les portent une identité bien précise. En (7b) donc, le poncho, les sombreros et les costumes des gitans aux couleurs vives transmettent une dimension socioculturelle espagnole.

À la lumière de tout ce qui précède, une question surgit : les deux books de Zorro sont-ils différents ? Oui... et non. La réponse est un peu normande, mais nous allons expliquer pourquoi. Non, ils ne sont pas nécessairement différents, car ils racontent tous les deux la même histoire, mettent en scène les mêmes personnages principaux et insèrent les chansons phares des Gipsy Kings. Pourtant ils diffèrent à des degrés fort divers. Les personnages jouant un rôle mineur ont tout bonnement été supprimés, la relation entre le protagoniste et l'antagoniste a été réécrite afin de répondre aux exigences culturelles du pays d'accueil et l'utilisation de l'espagnol a subi des traitements différents en fonction de la langue cible. Même si les stratégies d'écriture de Clark entraînent une perte au niveau du dépaysement interne, elles s'adaptent parfaitement à la langue du pays d'arrivée. Dans tous les cas de figure, le librettiste navigue en eaux troubles et doit sans cesse réécrire le book en fonction de la scène qui l'accueille. II effectue une espèce de refonte puisqu'il défait et recrée le book de 2008 pour que l'histoire du justicier masqué perdure. La réécriture du musical devient alors le lieu de changements qui, par extension, augmentent l'écart entre des cultures indéniablement différentes. Nous terminerons notre réflexion par ceci : le revival n'est pas uniquement fait de pertes irrémédiables, il est au contraire une amélioration progressive du book initial.

adefacq@mail.usf.edu

Alice Defacq enseigne le français à la University of South Florida St. Petersburg à Tampa, en Floride. Elle est titulaire d'un doctorat en traductologie de l'Université Catholique de l'Ouest - Collège Doctoral d'Angers (2011). Elle s'intéresse spécialement à l'adaptation des musicals anglo-américains pour la scène française. Ses recherches et publications portent sur la traduction chantable, l'adaptation et la circulation des musicals en France et au Québec. 


\section{Bibliographie}

APTER Ronnie et Mark HERMAN, 2016, Translating for Singing, London, Bloomsbury.

BASSNETT Susan et André LEFEVERE, 1998, Constructing Cultures: Essays on Literary Translation, Clevedon (Angleterre), Multinlingual Matters.

DAVIES Eirlys E. et Abdelâli BENTAHILA, 2008, "Translation and Code Switching in the Lyrics of Bilingual Popular Songs ", in The Translator, 14, p. 247-272.

GAMBIER Yves, 2004, "La traduction audiovisuelle : un genre en expansion ", in Meta, 16, p. 1-11. GREGORY William, 2010, "Jouabilité : un concept indéfinissable, incontournable... traduisible ou intraduisible?", in Traduire, 222, p. 7-21.

ISRAËL Fortunato, 1991, "Traduction littéraire : l'appropriation du texte ", La liberté en traduction, Didier Érudition.

KOUSTAS, Jane, 1988, "Traduire ou ne pas traduire le théâtre ? L'approche sémiotique ", in TTR, 1, p. 127-138.

LOW Peter, 2017, Translating Song: Lyrics and Texts, New York (États-Unis), Routledge.

MOREAU Marie-Louise, 1997, Sociolinguistique. Concepts de base, Hayen (Belgique), Éditions Mardaga.

PÉRAN Bruno, 2011, "Le surtitreur et son surtitrage : une activité qui reste à définir ", in ŞERBAN Adriana, LAVAUR Jean-Marc, (dir.), Traduction et médias audiovisuels, Villeneuve-d'Ascq (France), Presses Universitaires de Septentrion, p. 157-170.

PERROUX Alain, 2009, La comédie musicale : mode d'emploi, Paris, L'Avant-Scène Opéra.

REGATTIN Fabio, 2004, "Théâtre et traduction : un aperçu du débat théorique ", in L'Annuaire théâtral : revue québécoise d'études théâtrales, 36, p. 156-171.

UBERSFELD Anne, 1996, Lire le théâtre I. Paris, Belin.

VAN HOOF Henri, 1989, Traduire l'anglais. Paris, Duculot. 


\section{Échanges par courrier électronique}

CLARK Stephen, 2016a, message du 12 avril.

-, 2016b, message du 24 avril.

-, 2016c, message du 26 septembre.

,- 2016d, message du 30 septembre.

MATSUDA Naoyuki, 2017a, message du 21 novembre.

-, 2017b, message du 28 novembre.

-, 2017c, message du 30 novembre.

-, 2018, message du 14 janvier.

TARAUD Éric, 2017, message du 05 mars.

-, 2018, message du 07 janvier. 